\title{
Accurate Estimation of Correlation and Capacity for Hybrid Spatial-Angular MIMO Systems
}

\author{
Juan F. Valenzuela-Valdés, Antonio M. Martínez-González, and David A. Sánchez-Hernández, Senior Member, IEEE
}

\begin{abstract}
In this paper, several novel functions for accurately estimating the correlation and the multiple-input-multiple-output (MIMO) capacity of combined spatial and true polarization diversity (TPD) schemes are proposed for the first time. Minimum error estimation of the correlation for a hybrid spatial-TPD linear scheme previously reported cannot be obtained by simple nonlinear least-square estimators, and the use of genetic algorithms (GAs) provides an accurate solution. Channel matrix coefficients and MIMO capacities are analyzed for the diverse optimization strategies employed, which aim to identify the best prediction of MIMO performance. The novel functions are used with previously reported results and validated for Rayleigh fading scenarios with isotropic scattering using a multimode-stirred chamber (MIMO Analyzer). An accurate prediction of high correlation values is concluded to be of extreme importance for the final MIMO performance estimation. This has been found to be particularly important for estimating MIMO capacity with achieved prediction accuracies of $1.1 \%$ at $\mathrm{SNR}=15 \mathrm{~dB}$. In contrast, the accurate prediction of low correlation values has been found to have a less-important effect on the final capacity-predicting performance.
\end{abstract}

Index Terms-Correlation functions, dipole, genetic algorithms (GAs), multimode chamber, multiple input-multiple output (MIMO).

\section{INTRODUCTION}

$\mathbf{T}$ HE correlation between different multiple-input-multipleoutput (MIMO) channels is typically used to predict communication performance. When it is assumed that the antennas have the same radiation pattern and the correlation among the receive antennas is independent of the correlation between the transmit antennas, this model is known as the Kronecker model. This simplification may be justified by the fact that only immediate antenna surroundings cause the correlation between array elements and have a negligible impact on the correlation observed between the elements of the array at the other end of the link. Some other models with cross-correlation assumptions are also available [1], [2]. While some works claim that the impact of channel correlation on MIMO capacity is

Manuscript received June 10, 2008; revised November 4, 2008, January 22, 2009, and March 16, 2009. First published April 21, 2009; current version published October 2, 2009. This work was supported in part by the Spanish National R\&D Program under Grant TEC2008-05811. The review of this paper was coordinated by Prof. K. Fukawa.

J. F. Valenzuela-Valdés is with EMITE Ing, Centro Europeo de Empresas e Innovación de Murcia, 30100 Murcia, Spain (e-mail: juan.valenzuela@emiteingenieria.es).

A. M. Martínez-González and D. A. Sánchez-Hernández are with the Departamento de Tecnologías de la Información y Comunicaciones, Universidad Politécnica de Cartagena, 30202 Cartagena, Spain (e-mail: david.sanchez@upct.es).

Color versions of one or more of the figures in this paper are available online at http://ieeexplore.ieee.org.

Digital Object Identifier 10.1109/TVT.2009.2021182 negligible when the two-element beamwidth is smaller than the angular spread [3], others have recently identified limitations to the accuracy provided by the correlation-based models [4]. A particularly important limitation is related to their ability to represent geometry-based statistical models [5]. In spite of these claims, the accuracy of the Kronecker model has been demonstrated to be really dependent upon how the model renders the different eigenvalues of the channel correlation matrix [6]. When there is no covariance between the departure and arrival angles, the transmitter and receiver have been shown to have independent correlation [4]. This happens, for example, in isotropic scatterings, where all the angles of arrival (AoAs) are possible. A good example is that of urban outdoor scenarios and indoor scenarios with a great number of scatters, such as trees or metallic walls in buildings [7]. In these wide AoA scenarios, which are also typically emulated in reverberation chambers [4], [7], the Kronecker model performs reasonably well [6].

The importance of correlation-based models and their ability to predict MIMO performance has recently been augmented by diagonally correlated MIMO channels, which show a higher ergodic capacity than that provided by independent identically distributed (i.i.d.) fading channels [8]. An important class of diagonally correlated channels is constituted by multiple polarization. In these channels, the absence of accurate correlation models for predicting the interaction between antennas separated by both spatial and angular distance poses additional emulating difficulties. An accurate model for predicting the correlation between two linearly polarized antennas separated by both a spatial distance and an angular inclination is not yet available in the literature. In a previous letter [9], an important equivalence between angular separation and spatial separation was found, and simple coarse correlation models for a hybrid spatial-true polarization diversity (TPD) system were accordingly developed. In [9], however, the correlation model results tend to suggest that the linear scheme overestimates the correlation for spatial separation, whereas the quadratic model underestimates the correlation. In this sense, a higher order combining scheme or the use of weighting factors on the polarization separation and spatial separation models could help increase the hybrid correlation model's accuracy. Obtaining an accurate correlation model for hybrid spatial-TPD systems for the first time was the motivation of this paper. This is done by using the possibilities outlined in the results in [9]. In doing so, we have also found that an accurate prediction of the high correlation values is more important for final capacity performance prediction. This hybrid correlation model could also be useful when predicting the correlation between diverse modes with nonidentical polarization states in a multimode 
single antenna. Likewise, in handset MIMO, a relatively large number of antennas may be concentrated on a small volume in the presence of the user, who considerably modifies the mutual coupling and the correlation performance [10], [11]. In fact, we have shown that the correlation properties may not be sufficient to accurately predict the MIMO performance when in the presence of the user or low-efficiency antennas [10], [11], but it certainly plays an important role in its prediction. Therefore, an accurate MIMO performance prediction requires a precise correlation property forecast [12]. In this sense, in a recent letter [9], we have proposed the use of a spatial-based correlation function that is able to predict the correlation between two dipoles with a spatial separation and arbitrary inclination angles between transmission and reception. The obtained accuracy, however, was low and found to be model dependent, and more research was envisaged at that time to increase the model's accuracy by employing weighting factors.

In this paper, we present a novel set of functions for the accurate prediction of the matrix correlation properties of dipoles in both arbitrary positions and inclinations with respect to each other. These accurate functions are not currently available in the literature. Accurate correlation predictions allow for an accurate MIMO capacity forecast of the associated systems. As previously stated, the results in [9] suggested some accuracy increase by using a linear combination of functions with some unknown optimum weighting factors. However, the results in [9] did not clearly identify the best weighting factors. Consequently, in this paper, these novel functions are obtained through the use of genetic algorithms (GAs), which aimed to identify optimum weighting factors of the polynomialbased hybrid correlation functions. The capacity is estimated for different Rayleigh fading handset scenarios with isotropic scattering, wherein the Kronecker model performs at its best, but the correlation model accuracy is challenged.

\section{Simple CORRELATION FunCtions}

The spatial correlation between two adjacent identical antennas can be found in [13]. The autocorrelation coefficients between two vertically polarized antennas are described in [14] and [15] and validated for isotropic environments in a reverberation chamber by [16]

$$
\zeta=\sin \left\lfloor z_{\text {spatial }}\right\rfloor /\left\lfloor z_{\text {spatial }}\right\rfloor
$$

where $z_{\text {spatial }}=2 \pi d_{\text {spatial }} / \lambda$, and $d_{\text {spatial }}$ is the interelement distance. In TPD schemes [17], a new correlation function may be employed by establishing an equivalence between the angular and spatial separation [9] by

$$
\zeta=\sin \left\lfloor z_{\text {angular }}\right\rfloor /\left\lfloor z_{\text {angular }}\right\rfloor
$$

where $z_{\text {angular }}=2 \pi d_{\text {angular }}$. For a limited number of receiving antennas and under isotropic Rayleigh fading scenarios, the angular separation $d_{\text {angular }}$ can be made equivalent to a spatial separation by [9]

$$
d_{\text {angular }}=\varphi_{i-j}^{\prime} / 180 \text {. }
$$

In this model, the angular difference between two consecutive dipoles is transformed to an angle of the first quadrant in

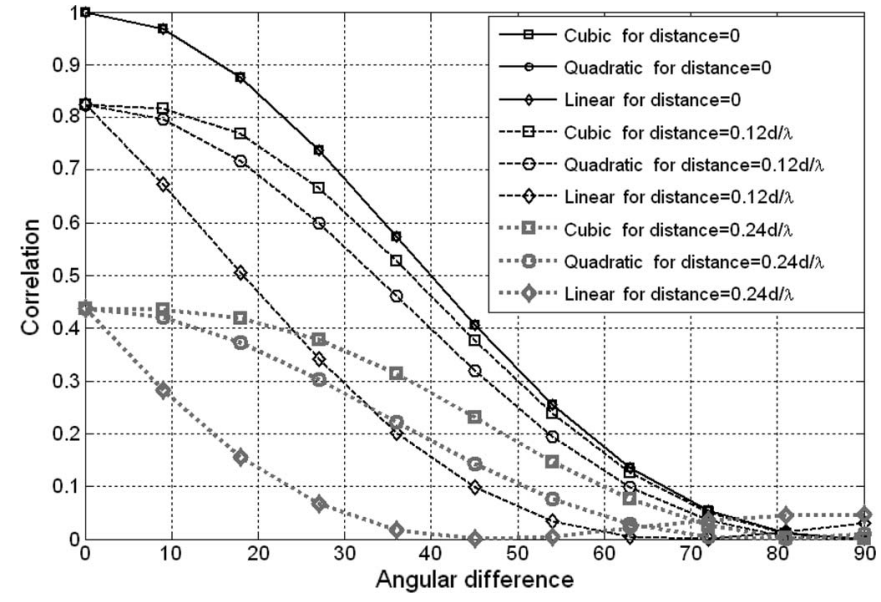

Fig. 1. Simple models for the combined correlation function versus angular difference with spatial separation as a parameter.

the following way:

$$
\varphi_{i-j}^{\prime}=\left\{\begin{array}{ccc}
a b s\left(\varphi_{i-j}\right), & \text { for } & a b s\left(\varphi_{i-j}\right) \leq 90 \\
180-a b s\left(\varphi_{i-j}\right), & \text { for } & 90 \leq a b s\left(\varphi_{i-j}\right) \leq 270 \\
360-a b s\left(\varphi_{i-j}\right), & \text { for } & 270 \leq a b s\left(\varphi_{i-j}\right) \leq 360
\end{array}\right\}
$$

where $\varphi_{i-j}=\varphi_{i}-\varphi_{j}$ is the angular difference between two dipoles in TPD schemes, and $\varphi_{i}$ and $\varphi_{j}$ are the orientation angles of dipoles $i$ and $j$, respectively. In [9], we proposed two simple linear and quadratic models to predict the correlation coefficients for the combined spatial and TPD schemes. The estimation error was not quantified in [9], but the two simple models had an opposite error behavior. This suggested the use of a linear combination of simple models to improve the model's accuracy. A third simple cubic model is presented in this paper. In all these simple models, a unique distance concept was employed by merging the spatial distance with its equivalent distance for angular diversity. The linear, quadratic, and cubic mergings are defined by

$$
\zeta=\frac{\sin \left[z_{\text {total } \_i}\right]}{\left[z_{\text {total } \_i}\right]} \quad \text { with }\left\{\begin{aligned}
& z_{\text {total } \_l}= z_{\text {spatial }}+z_{\text {angular }} \\
& z_{\text {total_q }}=\sqrt{z_{\text {spatial }}^{2}+z_{\text {angular }}^{2}} \\
& z_{\text {total_c }}=\sqrt[3]{z_{\text {spatial }}^{3}+z_{\text {angular }}^{3}}
\end{aligned}\right\} .
$$

The correlation coefficients for these models are depicted in Figs. 1 and 2. From Figs. 1 and 2, it is clearly observed that an equivalent correlation behavior is obtained for spatial separation and angular difference.

\section{Measurement Setup Error Estimation}

\section{A. Measurement Setup}

Validation measurements were carried out using the $8 \times 8$ MIMO Analyzer Series E100 by EMITE Ing in connection with the Rohde \& Schwarz ZVRE Vector Network Analyzer $(9 \mathrm{kHz}$ to $4 \mathrm{GHz}$ ). The MIMO Analyzer is a secondgeneration multimode-stirred chamber with dimensions of $0.82 \mathrm{~m} \times 1.275 \mathrm{~m} \times 1.95 \mathrm{~m}$, eight wall-mounted transmitting antennas, different holder-stirring positions, polarization 


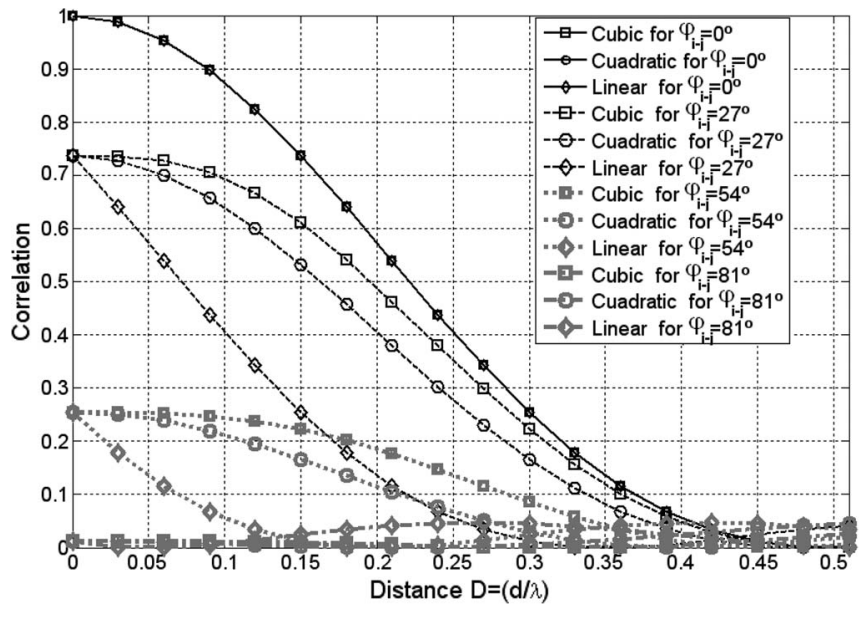

Fig. 2. Different models for the combined correlation function versus spatial separation with angular difference as a parameter.

stirring due to the different orientations of the wall-mounted printed-antenna exciting elements, two mechanical stirrers with 15 different positions for each platform position, 12 iriscoupling aperture stirring, and $20-\mathrm{MHz}$ frequency stirring. The measurements were performed at $900 \mathrm{MHz}$, and several half-wave dipoles were used as MIMO array receiving antennas. Despite the initial chambers being prepared to emulate the Rayleigh fading environments with some polarization imbalance through reverberation techniques, it has recently been demonstrated that both polarization performance [18] and Ricean fading scenarios [19] can properly be emulated in multimode-stirred chambers, which therefore extend their emulating capabilities indoors and to scenarios with a dominant line-of-sight component.

The emulated channel in the MIMO Analyzer was configured with that of an isotropic Rayleigh fading environment. Many authors have demonstrated that signal correlation degrades the MIMO performance. Consequently, correlation is typically used to estimate the MIMO capacity. However, other works have shown that the MIMO capacity also depends on the number of multipath components and the Rician k-factor [20]. As a consequence, in this paper, diverse measurement scenarios were prepared to evaluate all the ranges of spatial, angular, and combined correlation scenarios between dipoles. Tests were performed while trying to preserve the Rayleigh fading characteristic of the environment and the same number of multipath components. This was done by simply repeating exactly the same holder, polarization, frequency, iris, and mechanical stirrers' positions in all the tests. Different correlation coefficients were obtained by placing receive dipole number 1 in a fixed position and displacing receive dipole number 2. Dipole 2 was displaced a distance of $0.54 \lambda$ in $0.03 \lambda$ steps, while also rotating it $90^{\circ}$ relative to the position of receive dipole 1 at $9^{\circ}$ steps. The array setup is therefore a combined spatial and TPD scheme.

In the multimode-stirred chamber, the measured channel matrix $H_{r \times t}$ is defined as

$$
H_{r \times t}=R_{r}^{1 / 2} H_{r \times t}^{\mathrm{iid}}\left(R_{t}^{1 / 2}\right)^{T}
$$

where $r$ and $t$ indicate the number of receive and transmit antennas, $H_{r \times t}^{\mathrm{iid}}$ is a random matrix of complex Gaussian elements with i.i.d. zero mean and unit variance and dimensions $r \times t$, (.) ${ }^{T}$ denotes the transpose operation, and $R_{t}$ and $R_{r}$ are the separable transmit and receive spatial correlation matrices, respectively. In case some cross-correlation coefficients between the transmitter and receiver antennas exist, $H_{r \times t}$ is defined by [21], [22]

$$
H_{r \times t}=R_{r}^{1 / 2} H_{r \times s}^{\mathrm{iid}}\left(R_{s}^{1 / 2}\right) H_{s \times t}^{\mathrm{iid}}\left(R_{t}^{1 / 2}\right)^{T}
$$

where $R_{s}$ is the scattered cross-correlation matrix, and its dimensionality $s$ roughly corresponds to the number of scatters. Although heuristic in nature, this model has the advantage of an adequate modeling of rank-deficient channels, if necessary, through (7). The MIMO capacity can then be estimated with the well-known formula for Shannon capacity [22]

$$
C=\log _{2}\left(\operatorname{det}\left(I_{r}+\frac{\mathrm{SNR}}{t} H_{r \times t} H_{r \times t}^{\dagger}\right)\right)
$$

where $I_{r}$ is the identity matrix, and $(.)^{\dagger}$ denotes the transpose conjugate.

\section{B. Error Estimation}

To assess the goodness of the proposed correlation functions, we have employed the mean and maximum error values by

$$
\begin{aligned}
\operatorname{error}_{\text {mean }} & =\operatorname{mean}\left(\zeta_{\text {measured }}-\zeta_{\text {estimated }}\right) \\
\operatorname{error}_{\text {max }} & =\operatorname{mean}\left(\operatorname{abs}\left(\zeta_{\text {measured }}-\zeta_{\text {estimated }}\right)\right)
\end{aligned}
$$

in a way similar to that in [23], where $\zeta_{\text {measured }}$ is the matrix that contains all the measured correlation values in the multimode-stirred chamber, and $\zeta_{\text {estimated }}$ is the matrix that contains all the estimated correlation values using the proposed models. The obtained errors when estimating the correlation with the three simple models in the employed hybrid spatial and TPD scenarios are depicted in Fig. 3 and summarized in Table I.

It can be observed from Fig. 3 that the linear model underestimates the measured correlation, whereas the cubic model overestimates it. The simple quadratic model showed the best match out of all the simple models employed. It is important to point out, however, that the simple linear and cubic models inversely perform with respect to the quadratic model regarding the type of error being evaluated. A $120 \%$ deviation is observed for the linear model with respect to the quadratic model when evaluating the maximum error, whereas only an $81 \%$ deviation is found for the cubic model. However, this performance is inverted when the mean error is evaluated. In this case, a $40 \%$ deviation is experimented by the cubic model, and a $21 \%$ deviation is experimented by the linear model, which are both measured with respect to the quadratic model. A similar conduct with opposite maximum error behavior was found between the linear and quadratic models in [11], which, at that time, suggested the use of higher order functions. The results shown here demonstrate that accuracy is not improved by simply increasing the order of the estimating function. This, in turn, suggests that a minimum error estimation of the correlation 

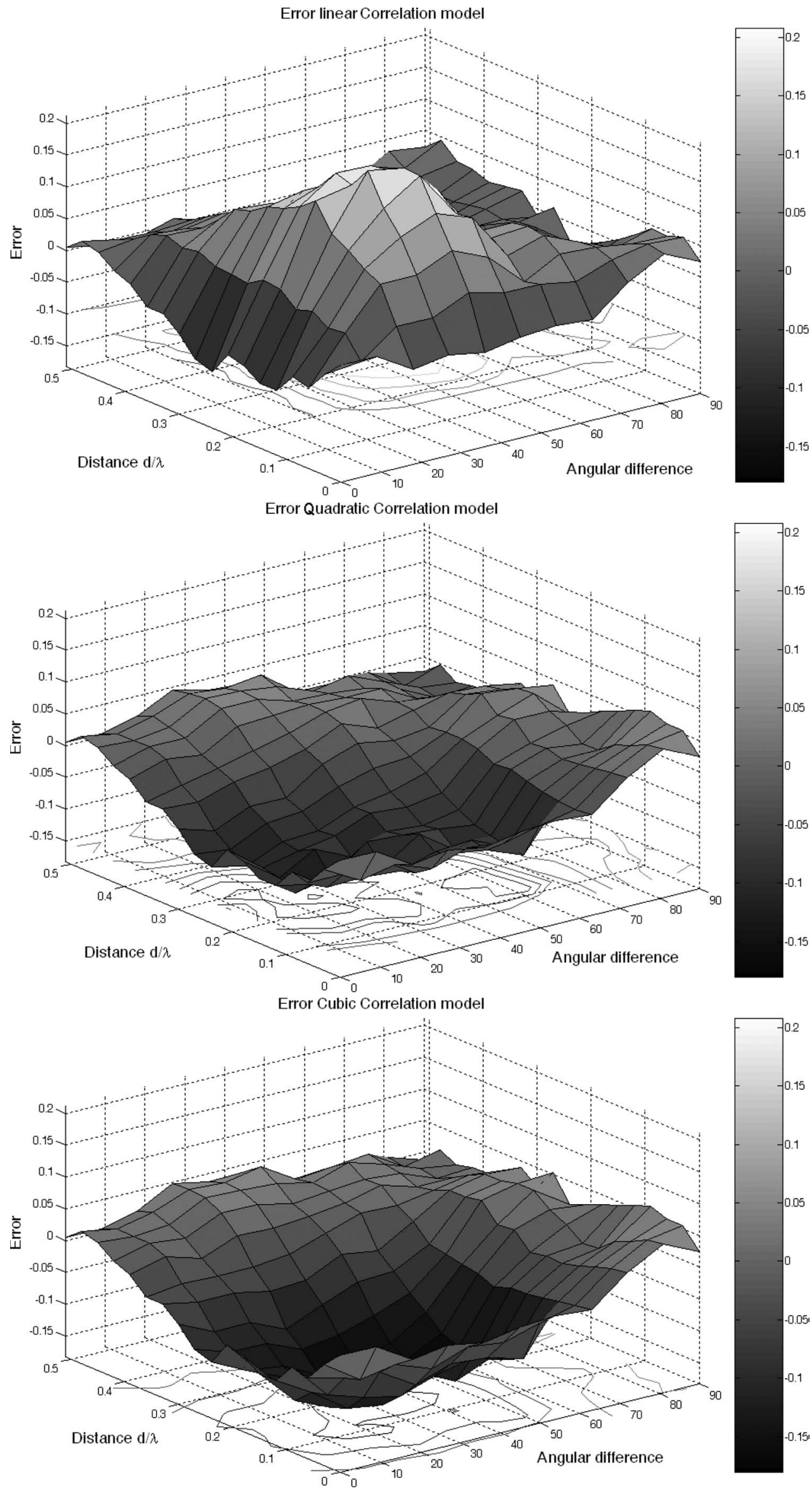

Fig. 3. Estimation errors for (top) linear, (middle), quadratic, and (bottom) cubic correlation combined functions. 
TABLE I

ERRORS FOR DiFFERENT SiMPLE CORRELATION MODELS

\begin{tabular}{lcc}
\hline \hline $\begin{array}{l}\text { Correlation } \\
\text { Model }\end{array}$ & Maximum Error & Mean Error \\
\hline Linear & 0.2112 & 0.0483 \\
Quadratic & 0.1008 & 0.0396 \\
Cubic & 0.1831 & 0.0555 \\
\hline \hline
\end{tabular}

for a hybrid spatial-TPD scheme may exist but cannot be obtained by simple nonlinear least-square estimators. In the next section, we have successfully employed a GA to weigh the three different combined functions for an optimum estimation model of the correlation that exists between two dipoles with arbitrary inclination angles and spatial distance between them. This is done in this paper in an accurate manner for the first time.

\section{GA OptimizATION}

The results in [9] showed that a weighted linear combination of the simple models could increase the accuracy. The fact that a simple increment on the order of the simple model does not increase the final predicting accuracy, as it has been demonstrated in the previous section, reinforces this previously reported conclusion. The proposed combined function is described by

$$
\begin{aligned}
z_{\text {total_optimum }}= & a^{*} z_{\text {total_linear }} \\
& +b^{*} z_{\text {total_quadratic }}+c^{*} z_{\text {total_cubic }}
\end{aligned}
$$

where $a, b$, and $c$ are unknown scalar values. The correlation estimates of this new function can be calculated in a similar way to that of simple functions by

$$
\zeta=\sin \left[z_{\text {total_optimum }}\right] /\left[z_{\text {total_optimum }}\right] .
$$

To find out the optimum values for $a, b$, and $c$, we have employed an in-house-programmed GA with different fitness functions [24], [25]. Each objective function to be minimized corresponds to a different strategy since the correlation is due to a complex hybridization between spatial and angular distances.

\section{A. Correlation-Based Optimization Strategies}

A simple minimization strategy would try to reduce either the mean or the maximum error defined by (9) with an associated fitness function defined by

$$
f_{\mathrm{st} 1}=\text { error }_{\text {mean }}, \quad f_{\mathrm{st} 2}=\text { error }_{\max } .
$$

However, it has been demonstrated that even with a correlation of $\sim 0.5$, almost total MIMO capacity can be obtained [26]. This means that the ability of the correlation function to estimate high values of correlation for the different possible situations is more important than the ability to accurately estimate low values of correlation. This is particularly important when the MIMO capacity is calculated from correlation values [3]. Consequently, two other fitness functions are proposed so that higher accuracy priority is given to high correlation values

\begin{tabular}{|c|c|c|c|c|}
\hline \multirow{2}{*}{ Scenario } & \multicolumn{2}{|l|}{ Receiver side } & \multicolumn{2}{|c|}{ Transmitter side } \\
\hline & $\begin{array}{l}\text { Spatial } \\
\text { distance }(d / \lambda)\end{array}$ & $\begin{array}{l}\text { Angular } \\
\text { Distance (o) }\end{array}$ & $\begin{array}{l}\text { Spatial } \\
\text { distance }(d / \lambda)\end{array}$ & $\begin{array}{l}\text { Angular } \\
\text { Distance }\left({ }^{o}\right)\end{array}$ \\
\hline $\bar{A}$ & 0.03 & 9 & 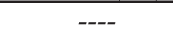 & ------ \\
\hline$B$ & 0.06 & 18 & ----- & ----- \\
\hline C & 0.09 & 27 & ----. & ------ \\
\hline$D$ & 0.03 & 9 & 0.03 & 9 \\
\hline E & 0.06 & 18 & 0.06 & 18 \\
\hline$F$ & 0.09 & 27 & 0.09 & 27 \\
\hline
\end{tabular}
when optimizing the function with GAs. Strategies 3.x were
TABLE II

Selected Evaluating Scenarios

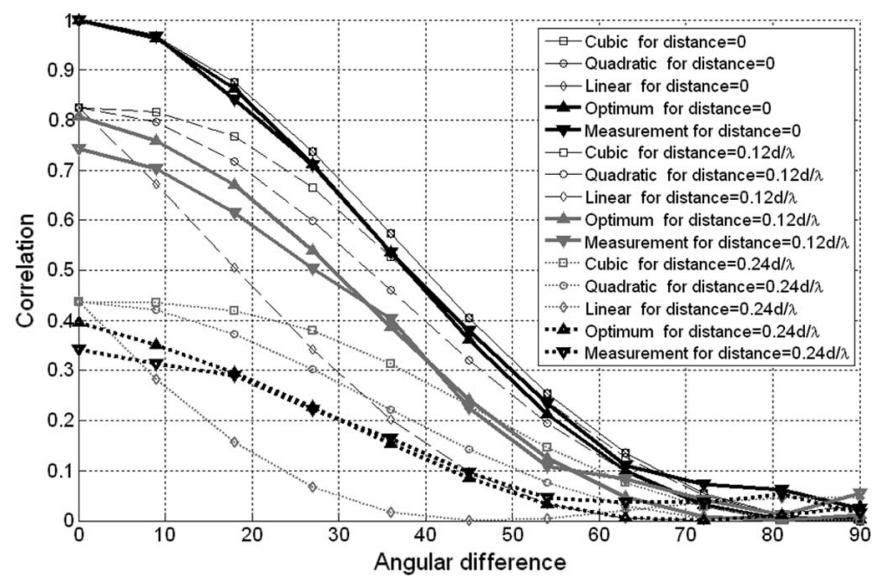

Fig. 4. Estimated results for diverse correlation functions and measured results for diverse MIMO scenarios with both spatial and angular distances.

designed to avoid evaluating errors within the fitness functions below a certain lower boundary for the correlation values by

$$
f_{\text {st3.x }}=\left\{\begin{array}{cl}
0 & \rightarrow 0 \leq \zeta_{\text {measured }} \leq \text { bound } \\
\left(\zeta_{\text {measured }}-\zeta_{\text {estimated }}\right) & \rightarrow \text { bound }<\zeta_{\text {measured }} \leq 1
\end{array}\right\}
$$

where $x$ indicates which lower boundary from 0.1 to 0.9 has been considered. Strategy 4 also has their priorities on the high correlation values but, this time, by weighting the fitness function in the following way:

$$
f_{\text {st4 }}=\left\{\begin{array}{cc}
\left(\zeta_{\text {measured }}-\zeta_{\text {estimated }}\right) & \rightarrow 0 \leq \zeta_{\text {measured }} \leq 0.5 \\
\left(\zeta_{\text {measured }}-\zeta_{\text {estimated }}\right)^{1 / 2} & \rightarrow 0.5<\zeta_{\text {measured }} \leq 0.7 \\
\left(\zeta_{\text {measured }}-\zeta_{\text {estimated }}\right)^{1 / 3} & \rightarrow 0.7<\zeta_{\text {measured }} \leq 1
\end{array}\right\} .
$$

\section{B. Capacity-Based Optimization Strategies}

We have recently made clear that the correlation does not tell the whole story when evaluating the MIMO capacity, particularly when the antenna efficiencies are taken into account or in the presence of the user [11]. Thus, a fifth strategy was designed to obtain the scalar values of the fitness functions for minimum MIMO capacity error when evaluated in all possible scenarios. The error in evaluating the MIMO capacity is defined by

$$
C_{\text {error }}^{\mathrm{SNR}}=\left(\left|\sum_{\mathrm{SNR}=0: 5: 35} C_{\text {measured }}^{\mathrm{SNR}}-C_{\text {estimated }}^{\mathrm{SNR}}\right|\right)
$$

where $C_{\text {estimated }}^{\mathrm{SNR}}$ is the estimated capacity for the evaluated scenario at different SNR values, and $C_{\text {measured }}^{\mathrm{SNR}}$ is the measured 


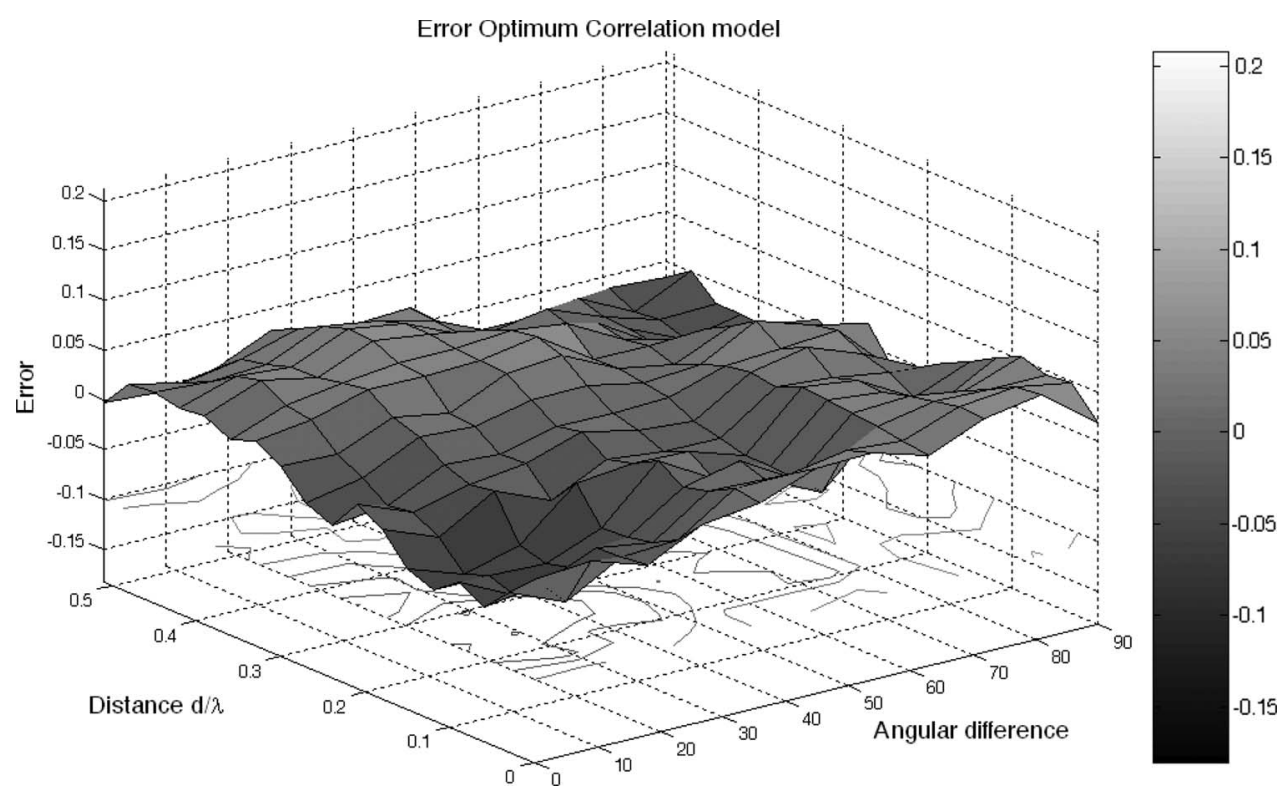

Fig. 5. Error for the GA simple mean error-optimized correlation function.

capacity for the evaluated scenario at different SNR values. The associated fitness function for the GA algorithm becomes

$f_{\mathrm{st} 5}=\sum_{x=A}^{x=F}\left(C_{\mathrm{error}}^{\mathrm{SNR}}=\left(\left|\sum_{\mathrm{SNR}=0: 5: 35} C_{\text {measured }}^{\mathrm{SNR}}-C_{\text {estimated }}^{\mathrm{SNR}}\right|\right)\right)$

where $A$ to $F$ indicate the selected scenario. A detailed description of scenarios A to $\mathrm{F}$ is provided in Table II. While looking at a final performance result, such as capacity, the convergence of the algorithm becomes slow in this strategy since, for each individual of each generation of each possible scenario at each possible SNR, capacity has to be estimated.

\section{RESULTS}

\section{A. Correlation Results}

A very good match between the correlation results obtained with the GA-optimized function with simple strategy 1 (identified as the optimum correlation model for the tested scenario) and the measured results was observed, as depicted in Fig. 4. In this figure, different correlation results are shown for several MIMO scenarios with both spatial and angular distances. The error results for this GA-optimized correlation function are illustrated in Fig. 5. This figure shows that improvements of over $35 \%$ with respect to the quadratic simple model results were obtained with simple GA-optimizing strategies. To make a comparison of the diverse GA-optimizing strategies, six different measurement scenarios were selected from those used in Fig. 4. Three selected scenarios had both TPD and spatial diversity and included both semicorrelated and correlated ends with high, medium, and low correlation values. A high correlation was employed by selecting low angular and spatial separation distances between dipoles. The medium- and low-correlation scenarios employed larger spatial and angular distances. The obtained errors when estimating the correlation model with the proposed methods with respect to measurements using the

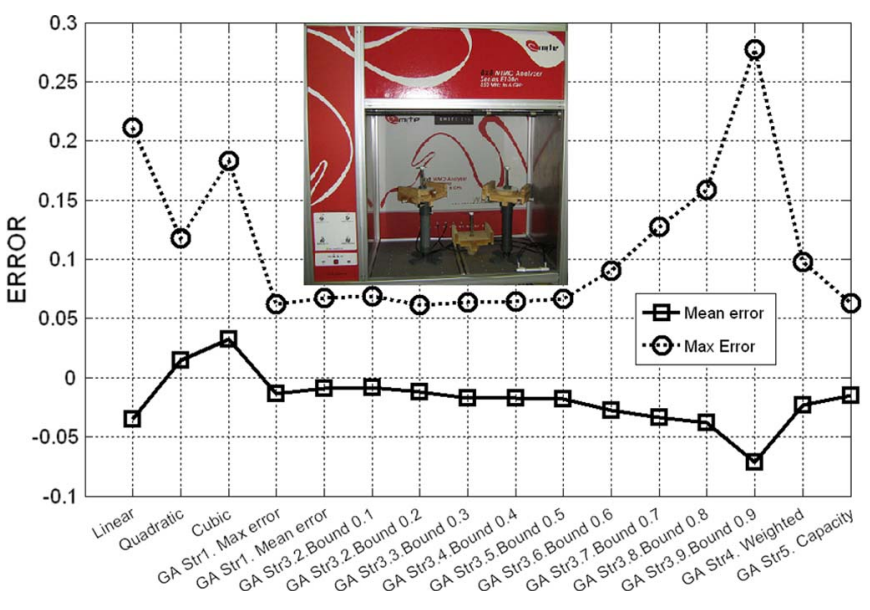

Fig. 6. Obtained errors when estimating correlation with the proposed models with respect to measurements using the $8 \times 8$ MIMO Analyzer.

$8 \times 8$ MIMO Analyzer are depicted in Fig. 6. A summary for the first three scenarios is extracted in Table III, wherein the mean and maximum obtained errors of the proposed models with respect to measurements are also listed.

It is interesting to note from Fig. 6 and Table III that the mean and maximum errors seem to have opposing behavior patterns. Likewise, when a bound is used in strategy 3 for GA optimization, the error increases with increasing correlation bound. Hence, using the bound to reduce the computational demands may not provide accurate prediction results. These results confirm our earlier finding of correlation being just part of the story for complicated real MIMO scenarios in reduced volumes [11]. The results shown in Table III are not conclusive for the selection of the GA-optimizing strategy and a call for additional metric evaluations for the new correlation functions.

\section{B. Channel Coefficients Results}

The way the proposed correlation function predicts not only the correlation factors, but some other parameters as well, 
TABLE III

ERRoRs IN DIFFERENT CORRELATION MODELS

\begin{tabular}{cccccc}
\hline \hline Model & $\begin{array}{c}\text { Coef } \\
\text { (a) }\end{array}$ & $\begin{array}{c}\text { Coef } \\
(\mathrm{b})\end{array}$ & $\begin{array}{c}\text { Coef } \\
(\mathrm{c})\end{array}$ & $\begin{array}{r}\text { Error } \\
\text { mean }\end{array}$ & $\begin{array}{r}\text { Error } \\
\text { max }\end{array}$ \\
\hline Linear & 1 & 0 & 0 & -0.035 & 0.211 \\
Quadratic & 0 & 1 & 0 & 0.015 & 0.118 \\
Cubic & 0 & 0 & 1 & 0.033 & 0.183 \\
GA Str1. Max error & 0.19 & 0.39 & 0.49 & -0.014 & 0.062 \\
GA Str2. Med error & 0.26 & 0.14 & 0.64 & -0.010 & 0.067 \\
GA Str3.1.Bound 0.1 & 0.24 & 0.12 & 0.70 & -0.008 & 0.069 \\
GA Str3.2.Bound 0.2 & 0.24 & 0.02 & 0.82 & -0.012 & 0.062 \\
GA Str3.3.Bound 0.3 & 0.27 & 0.07 & 0.74 & -0.017 & 0.064 \\
GA Str3.4.Bound 0.4 & 0.18 & 0.72 & 0.19 & -0.017 & 0.065 \\
GA Str3.5.Bound 0.5 & 0.55 & -0.78 & 1.29 & -0.018 & 0.067 \\
GA Str3.6.Bound 0.6 & 0.26 & 0.14 & 0.75 & -0.027 & 0.091 \\
GA Str3.7.Bound 0.7 & 0.33 & 0.79 & 0.01 & -0.033 & 0.128 \\
GA Str3.8.Bound 0.8 & 0.52 & 0.35 & 0.26 & -0.038 & 0.158 \\
GA Str3.9.Bound 0.9 & 0.29 & 0.364 & 0.82 & -0.072 & 0.278 \\
GA Str4. Weighted & 0.11 & 0347 & 0.69 & -0.024 & 0.098 \\
GA Str5. Capacity & 0.27 & 0.27 & 0.52 & -0.015 & 0.063 \\
\hline \hline
\end{tabular}
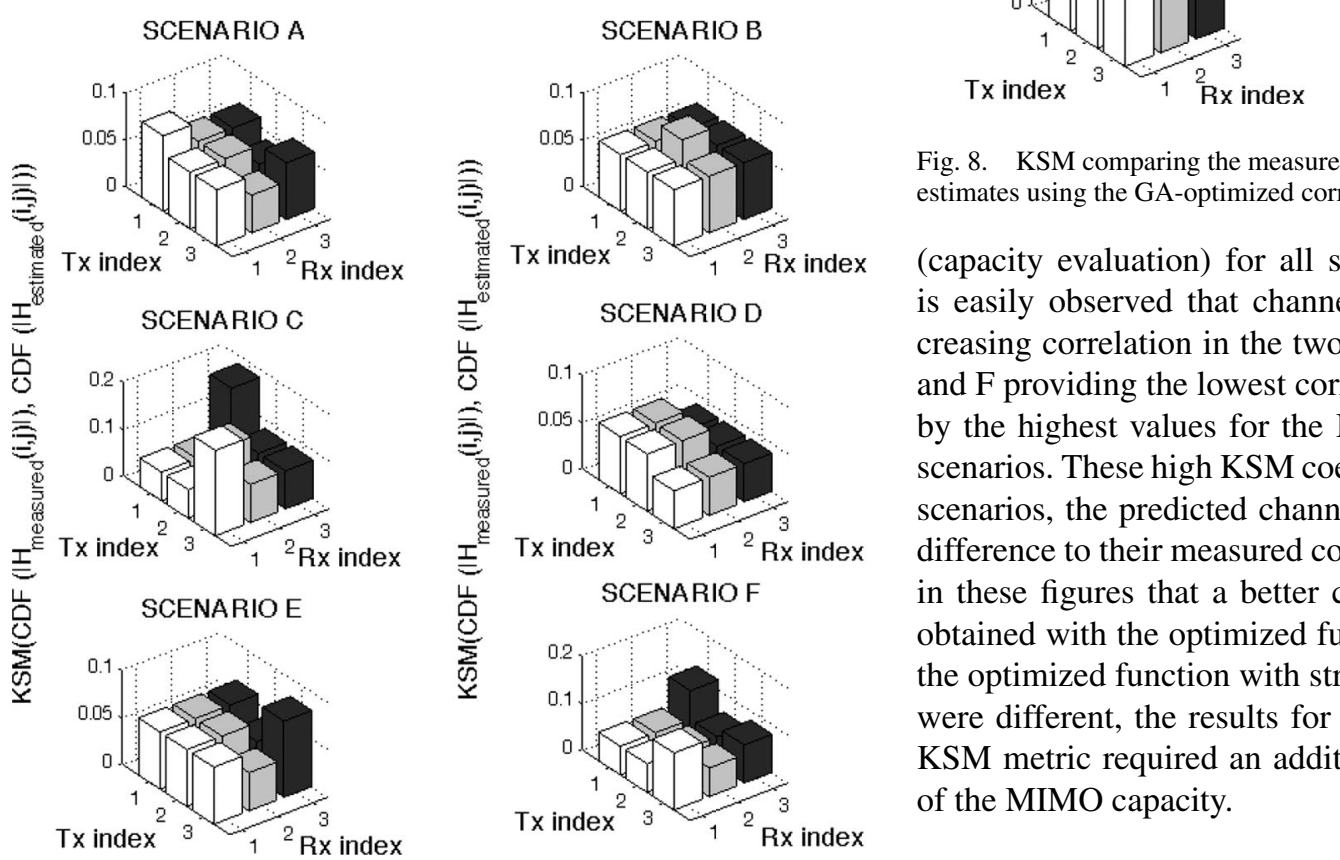
of the MIMO capacity.

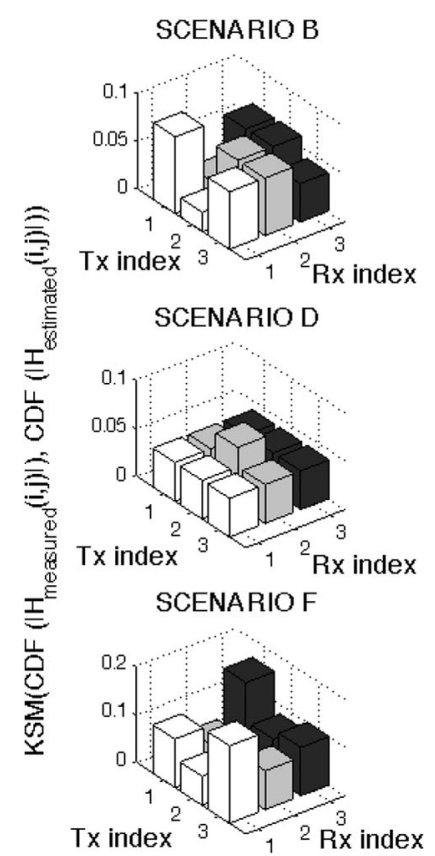

Fig. 8. KSM comparing the measured channel coefficient envelopes and their estimates using the GA-optimized correlation function with strategy 5.

(capacity evaluation) for all scenarios. From these figures, it is easily observed that channel estimation degrades with decreasing correlation in the two comparisons. With scenarios $\mathrm{C}$ and $\mathrm{F}$ providing the lowest correlation values, this is confirmed by the highest values for the KSM metric found in these two scenarios. These high KSM coefficients prove that for these two scenarios, the predicted channel coefficients attain the highest difference to their measured counterparts. Likewise, it is shown in these figures that a better channel-coefficient estimation is obtained with the optimized function with strategy 1 than with the optimized function with strategy 5 . Since the strategy goals were different, the results for the channel coefficient with the KSM metric required an additional analysis on the estimation

\section{MIMO Capacity Results}

Figs. 9 and 10 illustrate the estimated MIMO capacity values using simple correlation functions for scenarios $\mathrm{A}$ to $\mathrm{C}$ and D to F, respectively. The MIMO capacity was estimated from the predicted $\mathrm{H}$ matrix, which, in turn, was estimated from a Kronecker model using different correlation models described in this paper. The measured MIMO capacities for all scenarios are also plotted in Figs. 9 and 10 for comparison purposes. It can be observed from these figures that the linear model overestimates the measured capacity, whereas the cubic model underestimates it. The best fit within the simple correlation models is for the quadratic function, with scenario average capacity errors of $1.7 \%$ and $4.4 \%$ at $\mathrm{SNR}=15$ and $25 \mathrm{~dB}$, respectively. In all the models, the goodness of the estimation degrades with increasing SNR, which is consistent with the Kronecker model. A better goodness of fit is achieved by all GA-optimized functions in comparison with simple linear correlation functions, as depicted in Fig. 11 for scenarios A, with the GA-optimized correlation function using strategy 5 


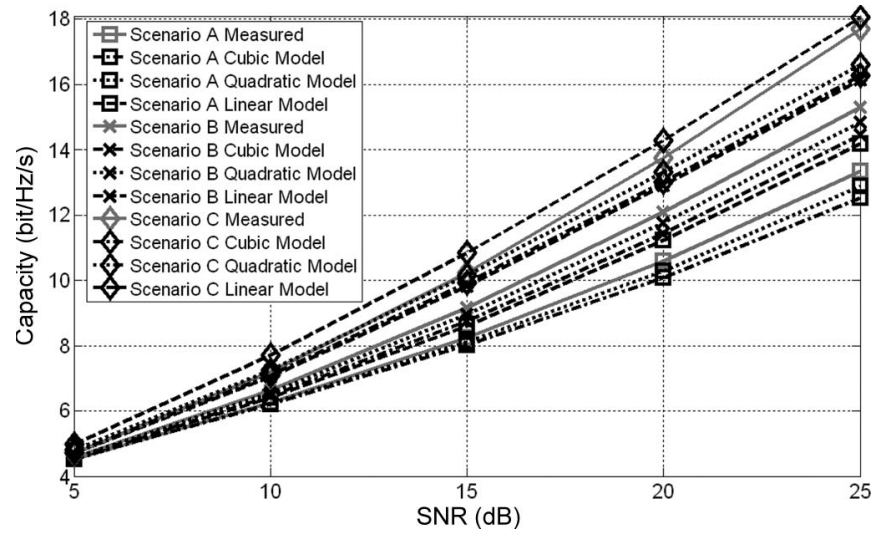

Fig. 9. Estimated MIMO capacities with simple correlation models for scenarios A to $\mathrm{C}$.

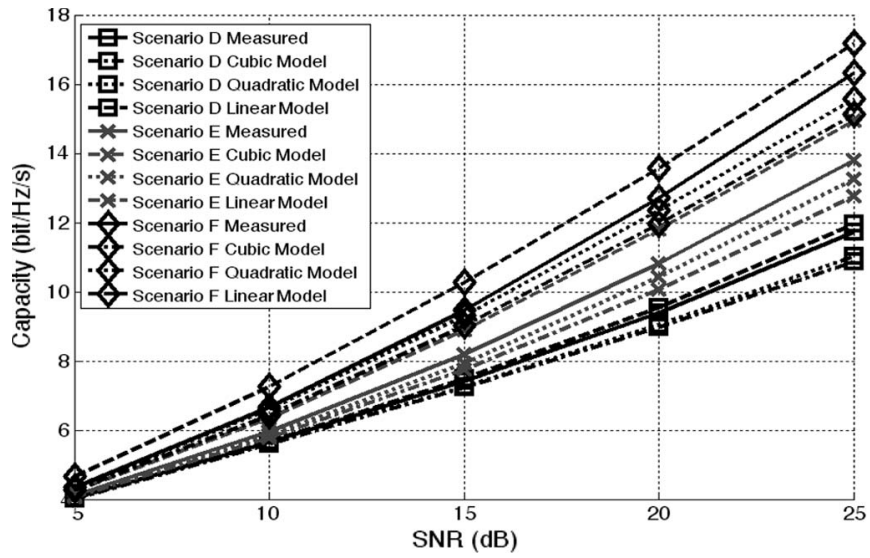

Fig. 10. Estimated MIMO capacities with simple correlation models for scenarios D to $\mathrm{F}$.

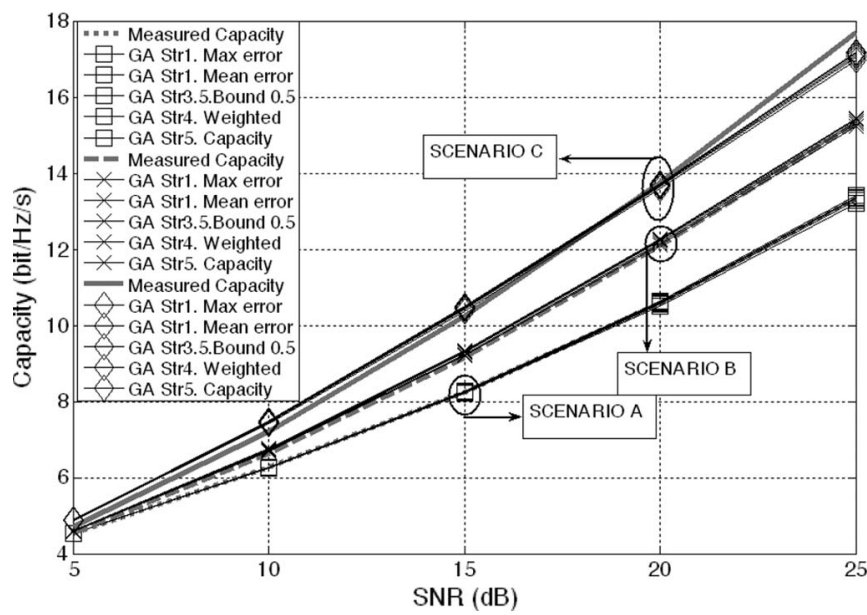

Fig. 11. Estimated MIMO capacities with GA-optimized correlation models for scenarios $\mathrm{A}$ and $\mathrm{C}$.

$\mathrm{B}$, and C. As it happened for the simple correlation models, the goodness of fit also degrades for an increasing SNR for the GAoptimized functions. The errors performed when estimating the MIMO capacity at low, high, and average SNR values are plotted in Figs. 12-14 for all GA-optimizing strategies and scenarios.

The worst predicting results are always obtained for scenarios $\mathrm{C}$ and $\mathrm{F}$, which attain the lowest correlation. It is also clear

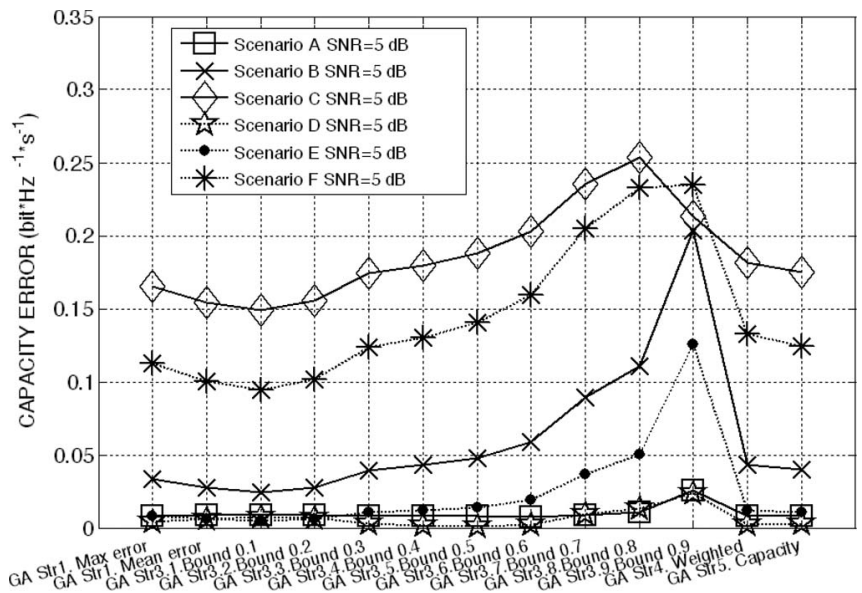

Fig. 12. Error when estimating MIMO capacities with GA-optimized correlation models at $\mathrm{SNR}=5 \mathrm{~dB}$.

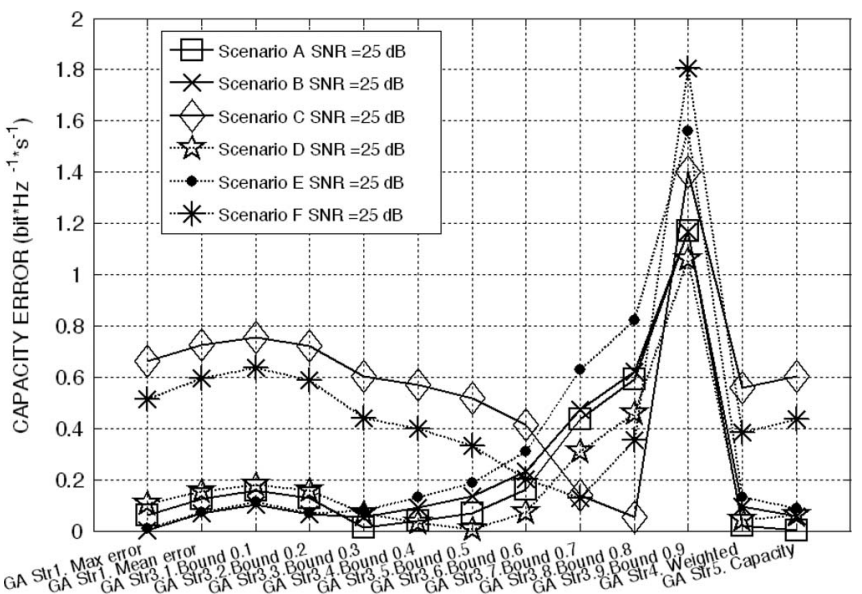

Fig. 13. Error when estimating MIMO capacities with GA-optimized correlation models at $\mathrm{SNR}=25 \mathrm{~dB}$.

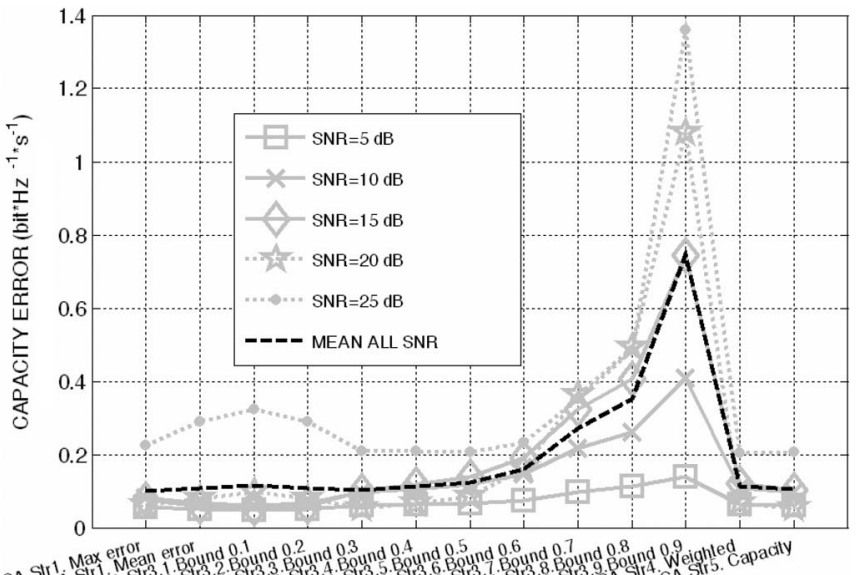

Fig. 14. SNR-averaged error when estimating MIMO capacities with GA-optimized correlation models.

from these figures that optimization strategies that ignore low correlation values work much better than the strategies that optimize either the maximum or the mean correlation error. It is, however, interesting to observe that the minimum SNRaveraged estimating error is provided for medium- to highcorrelated scenarios (A, B, D, and E) for the function with a 
bound optimizing limit of 0.5 . This agrees well with previous results, whereby this correlation value would not necessarily stop the MIMO designer from achieving quasi-optimum MIMO capacity [26]. This happens in spite of the fact that this optimization strategy provides higher mean and maximum error values than other GA optimization strategies. This is yet another confirmation that within a correlation model, the effort dedicated to accurately predict correlation values that are equal to or higher than 0.5 has to be more important than the effort dedicated to predict lower correlation values. It is equally interesting to see in Fig. 13 that the worst estimating performance corresponds to scenario $\mathrm{F}$, where both the transmitter and the receiver ends are low correlated. This was expected when observing the channel coefficient results. In Fig. 14, we can also extract which one is the GA-optimizing strategy that performs at its best when averaging all scenarios and SNRs. The best averaged MIMO capacity-predicting performance is attained by GA strategy 5 (capacity evaluation), as expected. This may overcome the largest computer resources demanded by this strategy. The novel proposed correlation model with this GA optimization strategy has scenario-average capacity errors of $1.1 \%$ and $1.3 \%$ at $\mathrm{SNR}=15$ and $25 \mathrm{~dB}$, respectively. The high accuracy of the proposed model is obtained at the cost of extra computational requirements.

\section{CONCLUSION}

In this paper, we have presented diverse correlation models for dipoles in isotropic scattering environments with Rayleigh fading. The novel GA-optimized correlation functions are able to accurately estimate the performance of complex combined spatial diversity and TPD schemes for the first time. In addition to the correlation values, channel matrix coefficients and MIMO capacity metrics have been presented. Through simulations and measurements, it has been demonstrated that a minimum error in the correlation function guarantees the minimum error in channel matrix coefficients. However, this does not guarantee a minimum error in MIMO capacity estimation, for which an accurate prediction of high correlation values is more important. The antenna elements investigated are dipoles with a single polarization only. This is helpful for using the model when predicting the correlation behavior of dual- or multiple-polarized antennas that are able to simultaneously capture several polarizations. Similarly, the model can be applied to large transmitting MIMO arrays or to receiver MIMO arrays at limited volumes and for arbitrary spatial separations and polarization states. This has been provided in this paper in an accurate manner for the first time. MIMO-capacity-predicting accuracies of $1.1 \%$ at $\mathrm{SNR}=15 \mathrm{~dB}$ have been achieved in the novel spatial-TPD MIMO scenarios. Future research includes the analysis of the model's accuracy for Rician fading and nonisotropic environments using the $8 \times 8$ MIMO Analyzer measuring capabilities.

\section{ACKNOWLEDGMENT}

The authors would like to thank the support of EMITE Ing personnel (www.emite-ing.com) and the Spanish Instituto de
Fomento de la Región de Murcia (Murcia’s Regional Development Agency).

\section{REFERENCES}

[1] D. Chizhik, F. Rashid-Farrokhi, J. Ling, and A. Lozano, "Effect of antenna separation on the capacity of BLAST in correlated channels," IEEE Commun. Lett., vol. 4, no. 11, pp. 337-339, Nov. 2000.

[2] A. Moustakas, H. Baranger, L. Balents, A. Sengupta, and S. Simon, "Communication through a diffusive medium: Coherence and capacity," Science, vol. 287, no. 5451, pp. 287-290, Jan. 2000.

[3] S. Loyka and G. Tsoulos, "Estimating MIMO system performance using the correlation matrix approach," IEEE Commun. Lett., vol. 6, no. 1, pp. 19-21, Jan. 2002.

[4] T. A. Lamahewa, R. A. Kennedy, T. D. Abhayapala, and T. Betlehem, "MIMO channel correlation in general scattering environments," in Proc. Australian Commun. Theory Workshop, 2006, pp. 93-98.

[5] C. Oestges, B. Clerckx, D. Vanhoenacker-Janvier, and A. J. Paulraj, "Impact of fading correlations on MIMO communication systems in geometry-based statistical channel models," IEEE Trans. Wireless Commun., vol. 4, no. 3, pp. 1112-1120, May 2005.

[6] A. A. Abouda, H. M. El-Sallabi, L. Vuokko, and S. G. Häggman, "Performance of stochastic Kronecker MIMO radio channel model in urban microcells," in Proc. 17th Annu. IEEE Int. Symp. PIMRC, 2006, pp. 1-5.

[7] Z. Yun and M. F. Iskander, "MIMO capacity for realistic wireless communications environments," in Proc. IEEE Antennas Propag. Soc. Int. Symp., Jun. 2004, pp. 1231-1234.

[8] H. Özcelik and C. Oestges, "Some remarkable properties of diagonally correlated MIMO channels," IEEE Trans. Veh. Technol., vol. 54, no. 6, pp. 2143-2149, Nov. 2005.

[9] J. F. Valenzuela-Valdés, A. M. Martínez-González, and D. SánchezHernández, "Estimating combined correlation functions for dipoles in Rayleigh-fading scenarios," IEEE Antennas Wireless Propag. Lett., vol. 6, pp. 349-352, 2007.

[10] J. F. Valenzuela-Valdés, A. M. Martínez-González, and D. SánchezHernández, "Effect of user presence on receive diversity and MIMO capacity for Rayleigh-fading channels," IEEE Antennas Wireless Propag. Lett., vol. 6, pp. 596-599, 2007.

[11] J. F. Valenzuela-Valdés, M. A. García-Fernández, A. M. MartínezGonzález, and D. Sánchez-Hernández, "The influence of efficiency on receive diversity and MIMO capacity for Rayleigh-fading channels," IEEE Trans. Antennas Propag., vol. 56, no. 5, pp. 1444-1450, May 2008.

[12] K. Yu and B. Ottersten, "Models for MIMO propagation channels: A review," Wirel. Commun. Mob. Comput., vol. 2, no. 7, pp. 653-666, 2002.

[13] Z. Tang and A. S. Mohan, "Characterize the indoor multipath propagation for MIMO communications," in Proc. APMC, Dec. 2005, vol. 4, pp. 2782-2785.

[14] R. H. Clarke, "A statistical theory of mobile radio reception," Bell Syst. Tech. J., vol. 47, no. 6, pp. 957-1000, 1968.

[15] W. C. Jakes, Microwave Mobile Communications. New York: Wiley, 1974.

[16] D. Hill and J. M. Ladbury, "Spatial correlation functions of fields and energy density in a reverberation chamber," IEEE Trans. Electromagn. Compat., vol. 44, no. 1, pp. 95-101, Feb. 2002.

[17] J. F. Valenzuela-Valdés, M. A. García-Fernández, A. M. MartínezGonzález, and D. Sánchez-Hernández, "The role of polarization diversity for MIMO systems under Rayleigh-fading environments," IEEE Antennas Wireless Propag. Lett., vol. 5, pp. 534-536, 2006.

[18] P. S. Kildal and C. Carlsson, "Detection of a polarization imbalance in reverberation chambers and how to remove it by polarization stirring when measuring antenna efficiencies," Microw. Opt. Technol. Lett., vol. 34, no. 2, pp. 145-149, Jul. 2002.

[19] C. L. Holloway, D. A. Hill, J. M. Ladbury, P. F. Wilson, G. Koepke, and J. Coder, "On the use of reverberation chambers to simulate a Rician radio environment for the testing of wireless devices," IEEE Trans. Antennas Propag., vol. 54, no. 11, pp. 3167-3177, Nov. 2006.

[20] C. Oestges, V. Erceg, and A. J. Paulraj, "A physical scattering model for MIMO macrocellular broadband wireless channels," IEEE J. Sel. Areas Commun., vol. 21, no. 5, pp. 721-729, Jun. 2003.

[21] D. Gesbert, H. Bolcskei, D. A. Gore, and A. J. Paulraj, "Outdoor MIMO wireless channels: Models and performance prediction," IEEE Trans. Commun., vol. 50, no. 12, pp. 1926-1934, Dec. 2002.

[22] H. Shin and J. H. Lee, "Capacity of multiple-antenna fading channels: Spatial fading correlation, double scattering, and keyhole," IEEE Trans. Inf. Theory, vol. 49, no. 10, pp. 2636-2647, Oct. 2003. 
[23] L.-B. Li and Z.-X. Wang, "A novel spatial correlation estimation technique for MIMO communication system," in Proc. 64th IEEE Veh. Technol. Conf., Sep. 2006, pp. 1-5.

[24] M. E. Requena-Perez, A. Albero-Ortiz, J. Monzó-Cabrera, and A. DíazMorcillo, "Combined use of genetic algorithms and gradient descent optimization methods for accurate inverse permittivity measurement," IEEE Trans. Microw. Theory Tech., vol. 54, no. 2, pp. 615-624, Feb. 2006.

[25] E. Dominguez-Tortajada, J. Monzó-Cabrera, and A. Díaz-Morcillo, "Uniform electric field distribution in microwave heating applicators by jeans of genetic algorithms optimization of dielectric multilayer structures," IEEE Trans. Microw. Theory Tech., vol. 55, no. 1, pp. 85-91, Jan. 2007.

[26] M. Kang and M.-S. Alouni, "Capacity of correlated MIMO Rician channels," IEEE Trans. Wireless Commun., vol. 5, no. 1, pp. 112-120, Jan. 2006.

[27] D. C. Boes, F. A. Graybill, and A. M. Mood, Introduction the Theory of Static, 3rd ed. New York: McGraw-Hill, 1974.

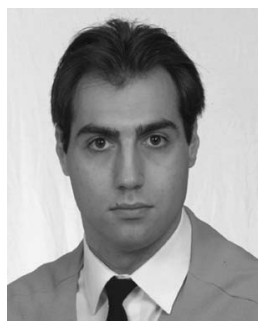

Juan F. Valenzuela-Valdés was born in Marbella, Spain. He received the degree in telecommunications engineering from the Universidad de Malaga, Malaga, Spain, in 2003 and the Ph.D. degree from the Universidad Politécnica de Cartagena, Cartagena, Spain, in May 2008.

In 2004, he was with CETECOM, Malaga. In 2004, he joined the Department of Information Technologies and Communications, Universidad Politécnica de Cartagena. He is currently the Head of Research with EMITE Ing, Centro Europeo de Empresas e Innovación de Murcia, Murcia, Spain. His current research areas cover multiple-input-multiple-output communications and SAR measurements.

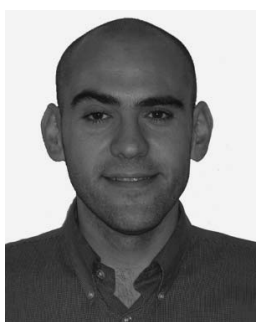

Antonio M. Martínez-González received the Dipl.Ing. degree in telecommunications engineering from the Universidad Politécnica de Valencia, Valencia, Spain, in 1998 and the Ph.D. degree from the Universidad Politécnica de Cartagena, Cartagena, Spain, in 2004.

From 1998 to September 1999, he was a Technical Engineer with the Electromagnetic Compatibility Laboratory, Universidad Politécnica de Valencia, where he developed assessment activities and compliance certifications with European directives related to immunity and emissions to electromagnetic radiation from diverse electrical, electronic, and telecommunication equipment. Since September 1999, he has been an Assistant Lecturer with the Universidad Politécnica de Cartagena. His research interest is focused on electromagnetic dosimetry and radioelectric emissions.

Dr. Martínez-González's works have been awarded with the Spanish National Prize from Foundation Airtel and from the Colegio Oficial de Ingenieros de Telecomunicación de España for the best final project on Mobile Communications in 1999.

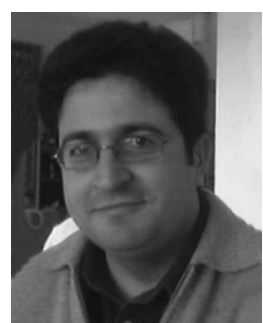

David A. Sánchez-Hernández (M'00-SM'06) received the Dipl.-Ing. degree in telecommunications engineering from the Universidad Politécnica de Valencia, Valencia, Spain, in 1992 and the Ph.D. degree from the University of London, London, U.K., in 1996.

From 1992 to 1994, he was a Research Associate with The British Council-CAM, King's College London, where he worked on active and dual-band microstrip patch antennas. In 1994, he was appointed as a European Union Research Fellow with King's College London, where he worked on several joint projects at 18,38 , and $60 \mathrm{GHz}$ related to printed and integrated antennas on GaAs, microstrip antenna arrays, sectorization, and diversity. In 1997, he returned to the Universidad Politécnica de Valencia, where he was a coleader of the Antennas, Microwaves, and Radar Research Group and the Microwave Heating Group. In early 1999 , he received the Readership from the Universidad Politécnica de Cartagena, Cartagena, Spain, where he was appointed the Vice Dean of the School for Telecommunications Engineering and leader of the Microwave, Radiocommunications, and Electromagnetism Engineering Research Group. In late 1999 , he was appointed Vice Chancellor for Innovation and Technology Transfer, Universidad Politécnica de Cartagena, as well as a member of several foundations and societies for the promotion of research and development (R\&D) in the Autonomous Region of Murcia, Murcia, Spain. In May 2001, he was appointed the Official Advisor of technology transfer and a member of The Industrial Advisory Council of the Autonomous Government of the Region of Murcia, and in May 2003, he was appointed the Head of Department. He has published several books, more than 40 scientific papers, and more than 80 conference contributions and is a Reviewer for several international journals and research programs. He is the holder of six patents. His current research interests encompass all aspects of the design and application of printed and integrated antennas for mobile communications, microwave heating, electromagnetic dosimetry issues, and mulitiple-input-multiple-output techniques for wireless communications.

Dr. Sánchez-Hernández is a Chartered Engineer. He is a Fellow Member of the Institution of Engineering and Technology, a member of the Ampere Board, and a member of the Comité Européen de Normalisation Electrotechnique TC106X. He is the recipient of the R\&D J. Langham Thompson Premium Award from the Institution of Electrical Engineers, as well as other national and international awards. 